\title{
16S rDNA microbiome composition pattern analysis as a diagnostic biomarker for biliary tract cancer
}

\author{
Huisong Lee ${ }^{1}$, Hyeon Kook Lee ${ }^{1 *}$ D, Seog Ki Min ${ }^{1}$ and Won Hee Lee ${ }^{2}$
}

\begin{abstract}
Background: The aim of this study is to investigate the composition of microbiota in biliary tract cancer patients and healthy adults by metagenome analysis and evaluate its potential values as biomarkers for biliary tract cancer.

Methods: Patients who were diagnosed with biliary tract cancer or benign inflammation were enrolled in this study. The control group consisted of healthy adults who presented with no history of significant medical issues. We isolated bacteria-derived extracellular vesicles in the plasma. The microbiome composition was investigated with 165 rDNA metagenome analysis. We evaluated each microbiome to ensure suitability for the biliary tract cancer prediction model.

Results: A total of 155 patients were included in this study: 24 patients with diagnosed biliary tract cancers, 43 diagnosed with cholecystitis or cholangitis, and 88 healthy adults. The microbiome composition pattern of the biliary tract cancer differed from the microbiome composition pattern seen in healthy adult group in beta diversity analysis. The percent composition of microbiota was found to be different from the phylum to genus level. Differences in the composition of the Bifidobacteriaceae and Pseudomonaceae families and Corynebacteriaceae Corynebacterium, Oxalobacteraceae Ralstonia and Comamonadaceae Comamonas species may be used to develop predictive models for biliary tract cancer.
\end{abstract}

Conclusion: Biliary tract cancer patients have altered microbiome composition, which represents a promising biomarker to differentiate malignant biliary tract disease from normal control group.

Keywords: Metagenome, Microbiota, Gallbladder neoplasms, Biomarkers, Extracellular vesicles

\section{Introduction}

Malignancy of the biliary tract is uncommon. However, it has poor prognosis for long-term survival. The prognosis differs depending upon the location and extent of disease [1]. For the treatment of biliary tract cancer, radical resection is necessary to improve survival outcomes [2]. However, the actual pathogenesis is not well understood. The chronic inflammation and liver fluke or typhoid fever have been thought to be a major cause of malignancy [3-7]. Recent reports suggest that certain bacteria, such as helicobacter, are associated with the development of gallbladder cancer $[8,9]$. Nevertheless, it is

\footnotetext{
*Correspondence: leehnkk@gmail.com

'Department of Surgery, Ewha Womans University College of Medicine, 1071

Anyangcheon-ro, Yangcheon-gu, Seoul 07985, South Korea

Full list of author information is available at the end of the article
}

unclear how the biliary tract cancer is related to the normal flora of our body. The microenvironments from normal flora can influence each other and even contribute to biliary tract cancer. Recently, techniques for meta-genome analysis have been rapidly developed. We can now analyze the microbiome from normal flora, which is considered to be important risk factors for genetic alteration of human $[4,5,10-12]$.

Previous studies were focused on parasite or viral infection for hepatobiliary malignancies. However, recent studies emphasize the fact that there exists another element of human development, arising from humanmicrobiome interaction. The microbiota constantly influences human cells and even provides opportunities to refine prenatal and postnatal growth [13]. The microbiome produces fluent of bacteria-derived extracellular

(C) The Author(s). 2020 Open Access This article is distributed under the terms of the Creative Commons Attribution 4.0 International License (http://creativecommons.org/licenses/by/4.0/), which permits unrestricted use, distribution, and 
vesicles (EV). Bacteria can communicate with each other and human cell by the EVs, and it can be detected in the blood, urine, bile, and stool [14-16]. However, there is much room for error in performing genome analysis, and the quality of sample is important [12]. The EVs can maintain its shape for a long time and preserve bacteria-derived genomes. We can effectively analyze the composition of microbiota by filtering EVs [17]. In an in vivo study, EVs of $P$. panacis could infiltrated the gut barrier and moved to the target organs. Moreover, gut microbes influence host metabolic homeostasis and contribute to the pathogenesis of type 2 diabetes, which is characterized by insulin resistance [16].

\section{Microbiome composition as a novel biomarker}

Microbe-derived EVs might be causative factors of various diseases. Recently, it has been determined that the EVs can even penetrate the blood-brain barrier. And there are documented differences in microbiome composition between autism spectrum disorder patients and the control group [18]. Moreover, there are studies to substantiate that microbiota is associated with colorectal cancer [19]. However, microbiome from bacteria-derived EVs was not investigated for biliary tract malignancy. The aim of this study is to compare the differences of composition of microbiota by metagenome analysis from bacteria-derived EVs. We expect that the composition of individual microbiome might be a novel biomarker to predict biliary tract cancer.

\section{Methods}

\section{Subjects and plasma sample preparation} Inclusion and exclusion criteria

The patients were enrolled from a single tertiary hospital. This study complied with the Declaration of Helsinki and was approved by the Institutional Review Board of Ewha Womans University Mokdong Hospital (2017-07-031). Written informed consent was obtained from all patients before surgery including genetic analysis. The control group consisted of normal healthy adults who agreed with informed consent during health checkup. The control subjects had no history of malignant disease, nor any clinical findings suggestive of gastrointestinal problems or neuropsychiatric disorders. The control subjects of this study had not taken antibiotics, probiotics, or prebiotics in the 3 months immediately antedating the sample collection.

Patients undergoing surgery for benign inflammation or malignant biliary tract disease were assessed for the study. Patients were included if they were 20 or more years of age and had no history of cognitive dysfunction to interfere with informed consent. If any patient was found to have a previous history of cancer or a Karnofsky performance scale of less than 70, that patient would be automatically excluded [20].
The patients were divided into three groups: biliary tract cancer, benign inflammation, and a control group. The patients who are diagnosed with cholecystitis or cholangitis, based upon documented evidence of pathology, were classified into the benign inflammation group.

\section{Sample collection}

A trained and well-qualified data manager reviewed the pathologic diagnosis, which was confirmed by hepatobiliary pathologist. The blood samples were obtained using standard protocols. Blood samples were collected from the median cubital vein into Vacutainer tubes that contained EDTA tubes (BD, Franklin Lakes, NJ, USA). Then, the sample was centrifuged at $1500 \mathrm{~g}$ for $10 \mathrm{~min}$. The plasma was isolated and immediately preserved in a freezer.

\section{Metagenome pattern analysis \\ EVs isolation and DNA extraction from human plasma samples}

EVs in human plasma were isolated using the differential centrifugation method as described previously [21]. For the extraction of DNA in isolated EVs, $1 \mu \mathrm{g}$ (based on the protein amount) of the EVs was boiled at $100^{\circ} \mathrm{C}$ for $15 \mathrm{~min}$, and then it was centrifuged at $10000 \mathrm{~g}$ for 20 min. The quality and quantity of the DNA were measured using the QIAxpert (QIAGEN, Germany).

DNA was extracted from EVs in human plasma using a PowerSoil DNA Isolation kit (MOBIO, USA). Bacterial genomic DNA was amplified with 16S_V3_F (5' TCGTCGGCAGCGTCAGATGTGTATAAGAGACAGC CTACGGGNGGCWGCAG-3') and 16S_V4_R (5' GTCTCGTGGGCTCGGAGATGTGTATAAGAGACAG GACTACHVGGGTATCTAATCC-3') primers, which are specific for $\mathrm{V} 3-\mathrm{V} 4$ hypervariable regions of $16 \mathrm{~S} \mathrm{rDNA}$ gene [22]. The libraries were prepared using polymerase chain reaction (PCR) products according to MiSeq System guide (Illumina, USA) and quantified using a QIAxpert (QIAGEN, Germany). After PCR products were extracted and quantified, equimolar ratios from each mixture were pooled and sequenced on a MiSeq (Illumina, USA) according to the manufacturer's recommendations.

\section{Taxonomic assignment}

Raw pyrosequencing reads obtained from the sequencer were filtered according to the barcode and primer sequences using MiSeq (Illumina, USA). Taxonomic assignment was performed by profiling program MDx-Pro ver.1 (MD Healthcare, Korea). To select $16 \mathrm{~S}$ rDNAs, all the sequence reads were compared to the GREENGENES. Sequence reads that had a similar sequence with more than 100 bit score and less than $1.0 \mathrm{E}$ value were admitted as partial $16 \mathrm{~S}$ rDNA sequences. Taxonomy-based analyses were performed using GREENGENES database [23, 24]. 


\section{Sample size estimation and statistical analysis}

This is the first study for metagenome analysis to compare the differences of composition of microbiome between the microbiome of patients afflicted with diagnosed biliary tract cancer and those suffering from benign biliary tract disease. Therefore, we cannot estimate the exact sample size. A previous study, which investigated microbiome composition, enrolled 20 patients in the patient group [18]. The initial goal of this study was to enroll more than 20 patients with diagnosed biliary tract cancer, as well as in excess of 40 benign inflammation cases. The control group was matched to the biliary tract cancer group and benign inflammation group with regard to chronologic age and sex. We performed logistic regression analysis with a randomized sampling of $30 \%$ of patients from each group. In the univariate analysis, we selected the top 5 microbiomes which were statistically associated with biliary tract cancer. Also, we performed multivariate analysis and tried to derive a prediction model. And we tried to validate the model with validation set.

The categorical variables are presented as number (percentage) and compared with $\chi^{2}$ test. The continuous variables are presented as the mean $\pm \mathrm{SD}$ and were compared using a Kruskal-Wallis test or ANOVA test. A $p$ value $<0.05$ was considered statistically significant.

\section{Biliary tract cancer prediction model development}

To reduce the selection bias, patients in the model development set were randomly allocated into "training" and "validation" sets. Four fifth of cases were assigned to the training set, and the other cases were assigned for test set [25].

We sorted the individual microbiome in the order of proportion and investigated the differences between the biliary tract cancer group and control group to find out potential markers with microbiome percent composition analysis. Significant microbiome was evaluated from phylum to family level. For the selection of the biomarkers, we considered relative abundances of operational taxonomic units (OTUs) at genus level. All prediction models include age and sex as covariates. First, we selected the candidate biomarkers with $p$ value $<0.01$, fold change $>2$-fold, and the average of relative abundances as $>0.1 \%$. Next, we used the Akaike Information Criteria (AIC) to infer a microbiome that is likely to be a biomarker candidate as a step-by-step selection method that compares predictive models with variable numbers of variables. Finally, the diagnostics model was calculated with the logistic regression. The regression coefficient $(b)$ of the logistic regression model was regarded as the log odds ratio (OR).

$$
p=\frac{e^{\beta_{0}+\beta_{1} x_{1}+\cdots+\beta_{7} x_{7}}}{1+e^{\beta_{0}+\beta_{1} x_{1}+\cdots+\beta_{7} x_{7}}}
$$

We developed the biliary tract prediction model with the coefficients based on the training set. It was validated by receiver operating characteristic (ROC) curve and calculation of the area under the ROC curve (AUC) with the validation set.

\section{Results}

\section{Patient demographics}

An aggregate 25 samples of biliary tract cancer were investigated, and one case was excluded after quality control testing. And 45 samples of benign inflammation group were evaluated, and two cases were excluded due to contamination. For the control group, 88 normal healthy adults were matched to the benign inflammation and biliary tract cancer group. Within the biliary tract cancer group, there were seven cases of diagnosed gallbladder cancers, nine intrahepatic cholangiocarcinoma, and eight extrahepatic cholangiocarcinoma. The average age of the biliary tract group, benign inflammation group, and control group were $69.8 \pm 10.7$ years, $55.4 \pm 15.5$ years, and $54.4 \pm 12.8$ years old, respectively (Table 1 ).

\section{Differences of microbiome composition in bacteria- derived EVs}

We isolated the bacteria-derived EVs. Then, variable regions of the 16S rRNA genes were amplified by PCR. We were able to identify over 7000 OTUs by subsequent DNA sequencing analysis in each biliary tract cancer patient, benign inflammation patient, and each individual in the control group. Among the identified OTUs, we assigned 41 OTUs at the phylum level, 102 OTUs at the class level, 203 OTUs at the order level, 384 OTUs at the family level, and 939 OTUs at the genus level. There were no differences in the alpha diversity. Therefore, we could perform further quantitative analysis and compare the microbiome composition (Fig. 1).

According to the taxonomy-based analysis, there were differences in the microbiome composition in beta diversity. We performed the principal component analysis of microbiota diversity based on the weighted UniFrac distance and Bray-Curtis dissimilarity. According to the dot pattern, we were able to roughly categorize the groups. As a result of the dot pattern, the microbiome pattern of the biliary tract cancer group was different from that of the normal healthy

Table 1 Characteristics of patients

\begin{tabular}{cllll}
\hline Characteristics & $\begin{array}{l}\text { Biliary tract } \\
\text { cancer } \\
(n=24)(\%)\end{array}$ & $\begin{array}{l}\text { Benign } \\
\text { inflammation } \\
(n=43)(\%)\end{array}$ & $\begin{array}{l}\text { Control } \\
(n=88)(\%)\end{array}$ & $p$ value $^{*}$ \\
\hline Sex Male & $15(63)$ & $15(35)$ & $37(42)$ & 0.086 \\
Female & $9(37)$ & $28(65)$ & $51(58)$ & \\
$\begin{array}{l}\text { Age (years) } \\
\text { (mean } \pm \text { SD) }\end{array}$ & $69.8 \pm 10.7$ & $55.4 \pm 15.5$ & $54.4 \pm 12.8$ & $<0.001$ \\
\hline
\end{tabular}

SD standard deviation

${ }^{*}$ Chi-square test or Kruskal-Wallis test 


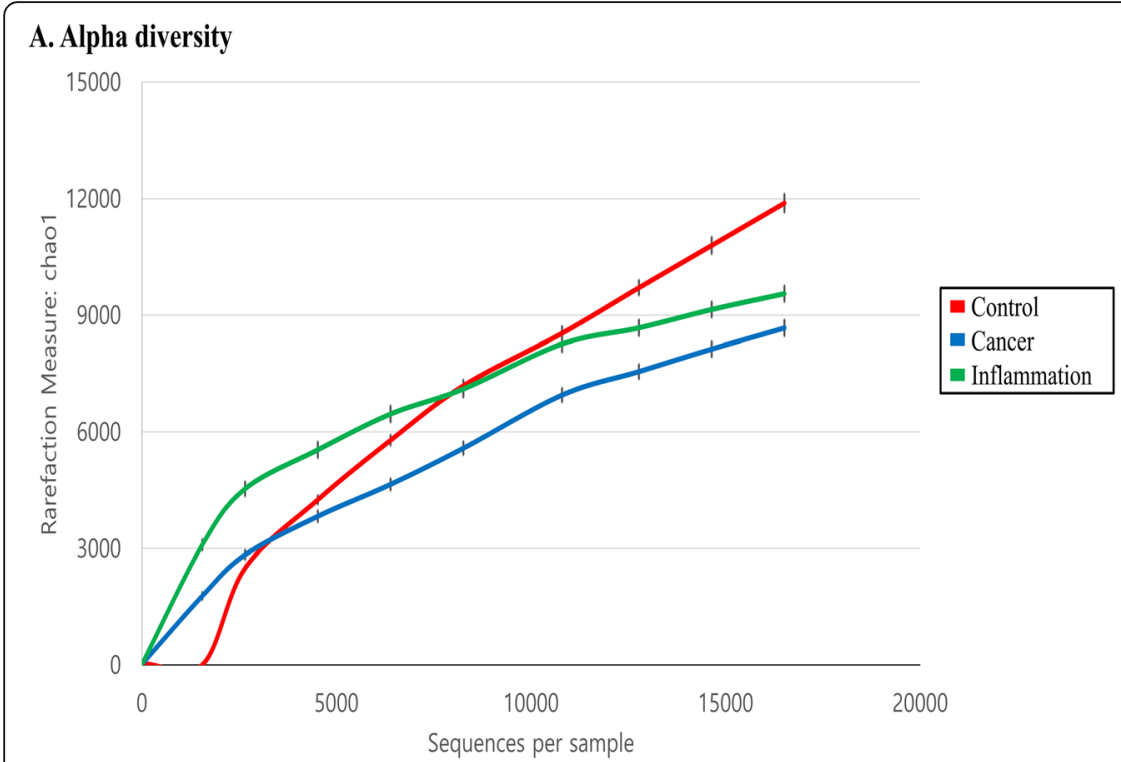

\section{B. Phylum level}

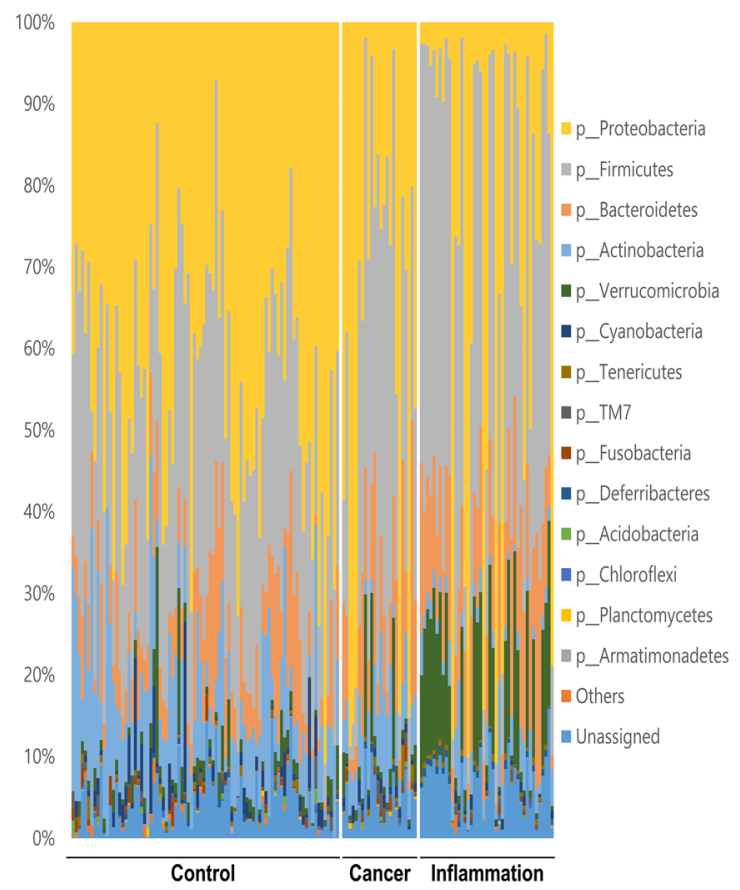

\section{Genus level}

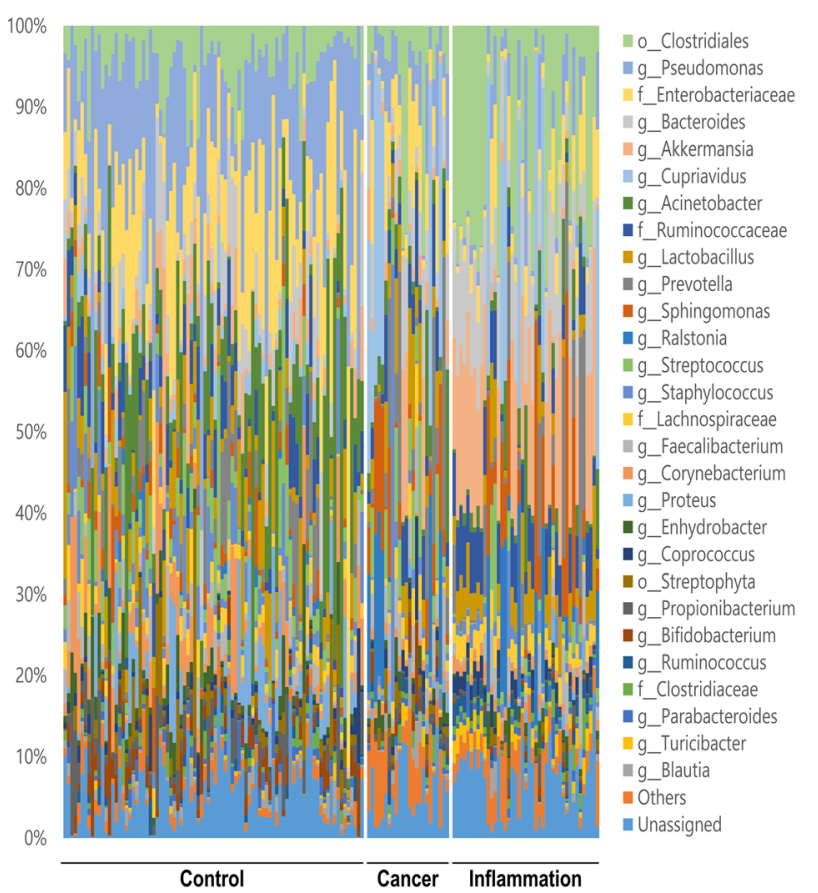

Fig. 1 a The alpha diversity curves. Rarefication curves representing the mean operational taxonomic units over the identified sequences of variable regions of $16 \mathrm{~S}$ rDNA gene in the biliary tract cancer, benign inflammation, and control group. b Microbiome composition analysis in phylum level. c Microbiome composition analysis in genus level

group, but similar to that of the cholecystitis group (Fig. 2). We analyzed the percent composition of individual microbiome from phylum to family level. Sequence readings of EVs-based $16 \mathrm{~S}$ rDNA indicated that the top five members of the phyla $p_{-}$Proteobacteria, p_Firmicutes, p_Actinobacteria, p_Bacteroidetes, and $p_{-}$Cyanobacteria comprised $94.7 \%$ of the identified OTUs in healthy subjects, whereas these members covered $93.8 \%$ of the total OTUs in the biliary tract cancer individuals. In the benign inflammation group, the proportion of top five phyla was $88.1 \%$ and lower than the other two groups. We could therefore surmise that the patients with cholecystitis or cholangitis have altered phyla composition. The occupancy of $p_{-}$Proteobacteria of the biliary tract cancer patients, benign inflammation patients, and in the control 


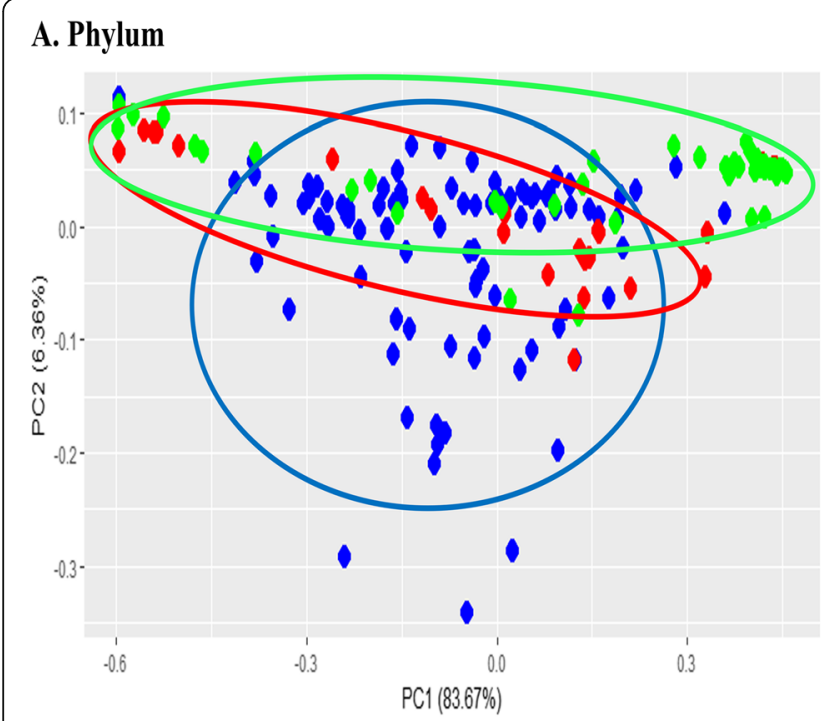

\section{- Biliary track cancer \\ - Benign inflammation \\ Control}

\section{Order}

\section{B. Class}
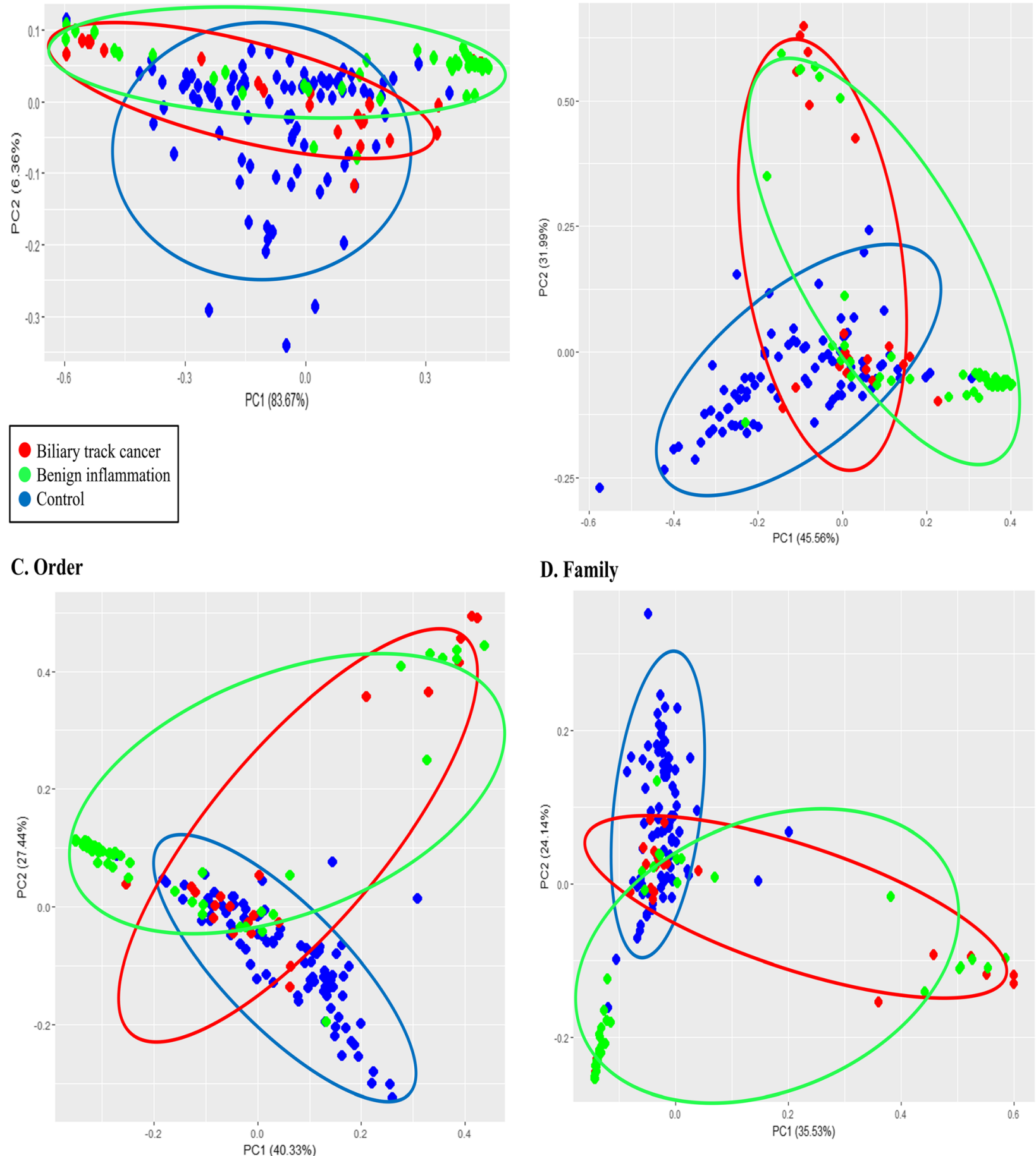

\section{Family}

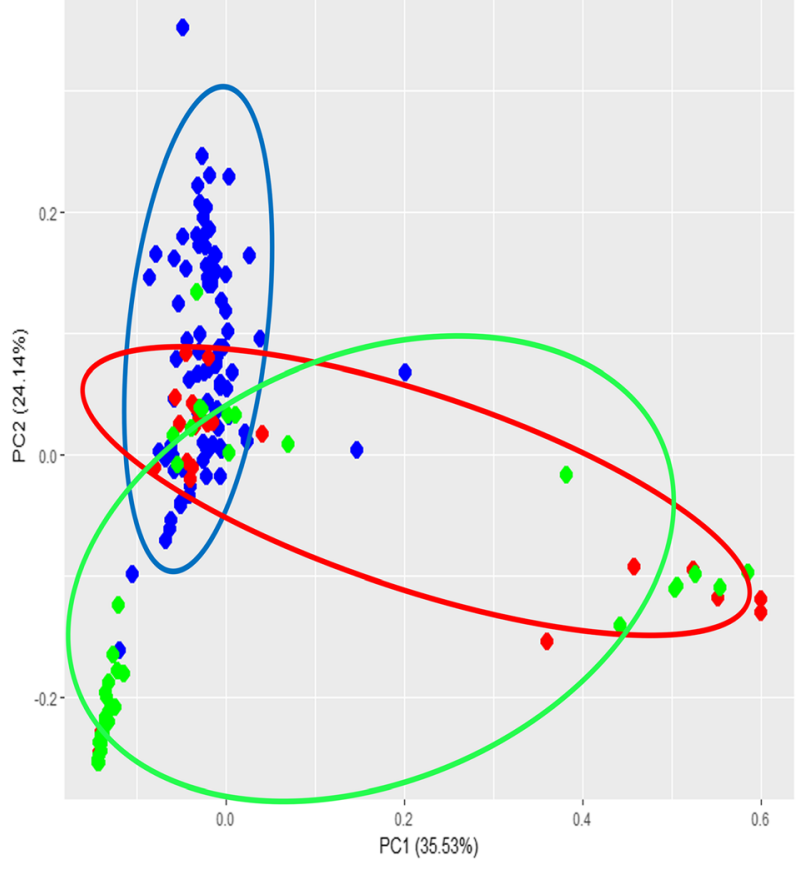

Fig. 2 The beta diversity sheet of metagenome pattern from the phylum to family level. Principal component analysis of microbiota diversity based on the weighted UniFrac distance and Bray-Curtis dissimilarity. Biliary tract cancer (red), benign inflammation (green), and control (blue). a Phylum level, b Class level, c Order level, d Family level

group was $38.4 \%, 30.0 \%$, and $43.9 \%$, respectively. On the other hand, the proportion of p_Firmicutes was 33.1\%, $39.1 \%$, and $28.2 \%$, respectively.
In class level analysis, the proportion of $c_{-}$Clostridia of biliary tract cancer, benign inflammation, and the control group was $30.1 \%, 19.9 \%$, and $15.1 \%$, respectively, although 


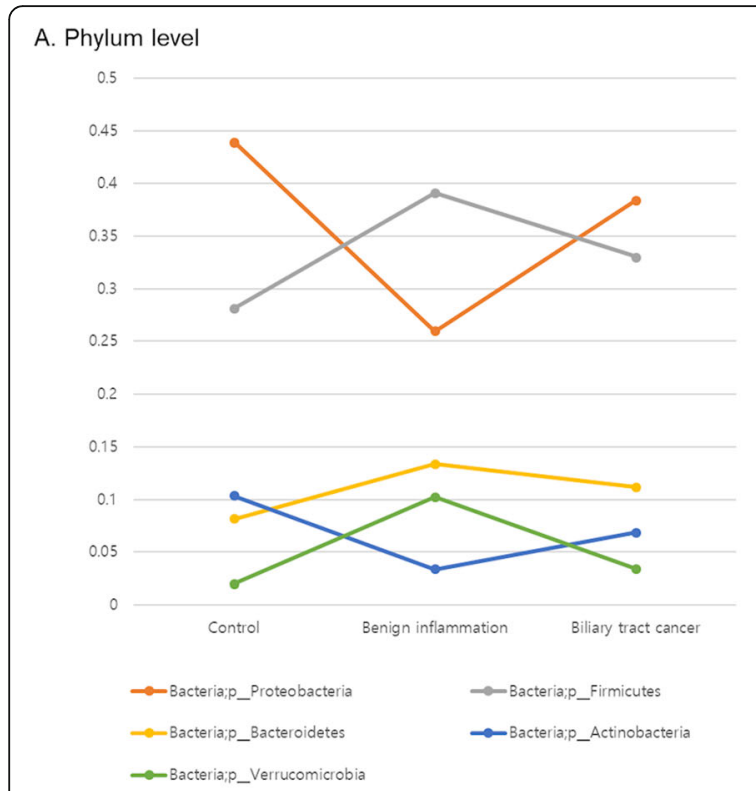

C. Order level

\section{B. Class level}
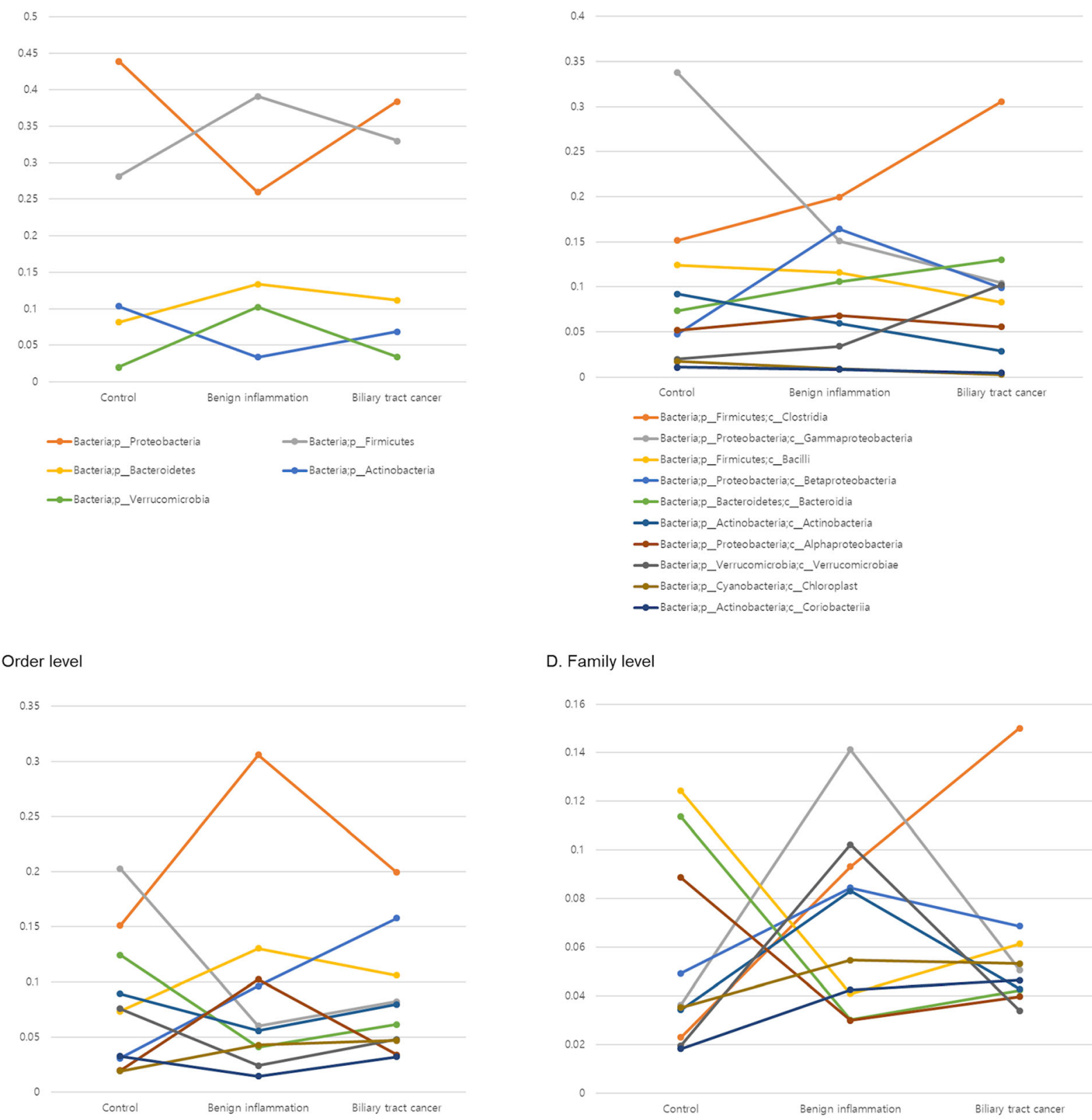

D. Family level

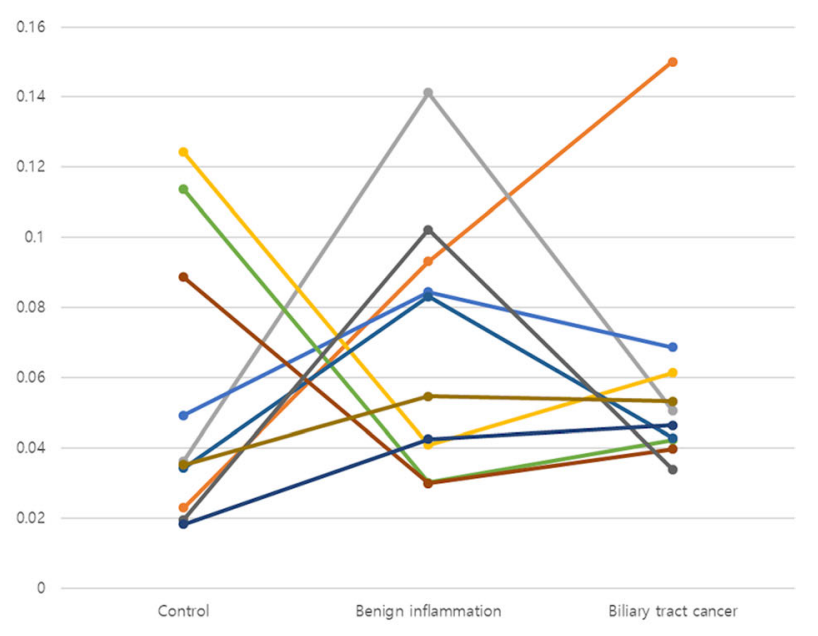

$\rightarrow$ Bacteria;P_Firmicutes;C_Clostridia;o_Clostridiales

$\rightarrow$ Bacteria;P_Proteobacteria;c_Gammaproteobacteria;o_Pseudomonadales

$\longrightarrow$ —Bacteria;p_Bacteroidetes;c_Bacteroidia;o_Bacteroidales

$\rightarrow$ Bacteria;p_Proteobacteria;c_Betaproteobacteria;o_Burkholderiales

$\sim$ Bacteria;p_Proteobacteria;c_Gammaproteobacteria;o_Enterobacteriales

$\rightarrow$ Bacteria;p_Firmicutes;__Bacilli; __Lactobacillales

—Bacteria;p_Verrucomicrobia;c_Verrucomicrobiae; __Verrucomicrobiales

$\rightarrow$ Bacteria;p_Actinobacteria;C_Actinobacteria;O_Actinomycetales

$\rightarrow$ Bacteria;p_Proteobacteria;c_Alphaproteobacteria;o_Sphingomonadales

$\rightarrow$ Bacteria,p_Firmicutes;c_Bacilli;o_Bacillales

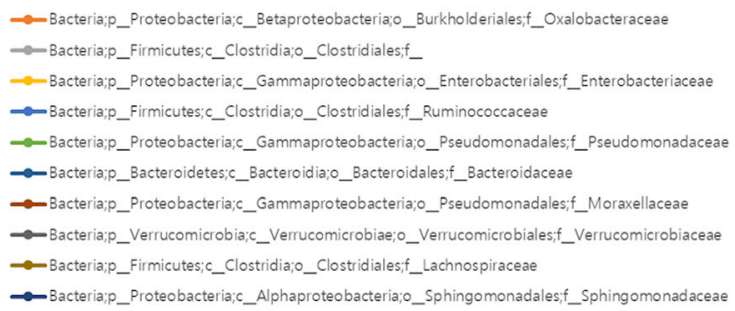

Fig. 3 The percent composition of microbiota from phylum to family level. Overall composition of microbiota was compared. The top five subjects in phylum level and top ten subjects from class to family level are presented. a Phylum level, b Class level, c Order level, d Family level 
the proportion of c_Gammaproteobacteria was 10.4\%, $15.1 \%$, and $33.8 \%$, respectively (Fig. 3 ).

\section{Biliary tract cancer prediction model}

We could identify five important microbiomes that the occupancy rate was significantly increased or decreased on the percent composition analysis. The compositional differences of Bifidobacteriaceae family and Oxalobacteraceae Ralstonia was found to be a significant positive marker, and the Pseudomonaceae family, Corynebacteriaceae Corynebacterium, and Comamonadaceae Comamonas species were found to be significant negative markers to differentiate biliary tract cancer patients from the individuals in the control group. We developed the biliary tract cancer prediction model with these five variables in company with chronologic age and sex based on the training set (Table 2).

We validated the prediction model with ROC curve, and the AUC was one. The composition of these five markers was obviously different upon comparison made between the biliary tract cancer patient and the control group. The accuracy was 1.0000 (confidence interval, 0.8518 to 1.0000 ), kappa value was 1.0000 , and $p$ value was 0.0035 . The sensitivity of the model was 1.0000 , and the specificity was 1.0000. The positive prediction value was 1.0000 , and the negative prediction value was 1.0000 . The balance accuracy was 1.0000 (Fig. 4a).

We compared the microbiome composition between the biliary tract cancer and cholecystitis groups. The composition of Bacillus and Bifidobacterium genus were different. In order level, Anaeroplasmatales, Erysipelotrichales, and Bacteroidales were different. However, there was no statistically significant difference in ROC curve analysis (Fig. 4b).

\section{Discussion}

The microbiota is thought to be a component of the human body and a source of genetic diversity and modifier of disease [11,26]. Moreover, recent studies investigated and revealed that there is extremely active interaction and synergistic effect between the microbiota and human host cells by EVs. This mechanism has been recently spotlighted as a pathogenetic mechanism of various diseases [14, 15, 27-29].

To the best of our knowledge, this study is the first attempt to analyze the composition of microbiome from EVs in patients with biliary tract cancer. Ultimately, we were able to successfully analyze the composition of microbiome in biliary tract cancer and benign inflammation patients compared with normal healthy adult group. We determined that specific families or species were extremely increased or decreased in the biliary tract cancer group, when compared to the control group. The compositional differences of Bifidobacteriaceae and Pseudomonaceae families and Corynebacteriaceae Corynebacterium, Oxalobacteraceae Ralstonia, and Comamonadaceae Comamonas species were found to be significant markers to make a biliary tract prediction model. In this study, however, the microbiome composition was similar between patients with cholecystitis and biliary tract cancer. Chronic inflammation such as chronic cholecystitis or cholangitis is also associated with cancer development. Previous studies have reported that Helicobacter species are associated with the development of both gallstones and gallbladder cancer $[9,30,31]$. Based on these results, it will be possible to use the microbiome pattern as a marker of cancer diagnosis in the future.

Traditionally, chronic cholecystitis or cholangitis is thought to be associated with malignant transformation [32]. In previous studies, certain bacteria were reported to be associated with the development of gallstone and biliary tract cancer $[8,9,30,31]$. However, the actual mechanism of transformation has not yet been identified. We believe that the metagenome analysis helps us to explain the actual pathogenesis by which inflammatory changes transform to progressive malignancy. The metagenome analysis from blood sample represents the altered microbiota composition. Previous studies have demonstrated, from the blood and urine, EVs which were partially consistent with the results from fecal samples $[18,33]$. We can explore and investigate the alteration of

Table 2 The biliary tract cancer prediction model. The compositional differences of Bifidobacteriaceae and Pseudomonaceae families and Corynebacteriaceae Corynebacterium, Oxalobacteraceae Ralstonia, and Comamonadaceae Comamonas species were significant positive or negative markers to differentiate biliary tract cancer from control group

\begin{tabular}{lll}
\hline X & Variables & B \\
\hline X1 & Age & 1.376 \\
X2 & Sex & -12.98 \\
X3 & P_Actinobacteria; C_Actinobacteria; O_Bifidobacteriales; f_Bifidobacteriaceae & 567,500 \\
X4 & P_Proteobacteria; C_Gammaproteobacteria; O_Pseudomonadales; f_Pseudomonadaceae & $-58,450$ \\
X5 & P_Actinobacteria; C_Actinobacteria; O_Actinomycetales; f_Corynebacteriaceae; g_Corynebacterium & -1943 \\
X6 & P_Proteobacteria; C_Betaproteobacteria; O_Burkholderiales; f_Oxalobacteraceae; g_Ralstonia & 2881 \\
X7 & P_Proteobacteria; c_Betaproteobacteria; O_Burkholderiales; f_Comamonadaceae; g_Comamonas & $-39,210$ \\
& Intercept & -18.35 \\
\hline
\end{tabular}




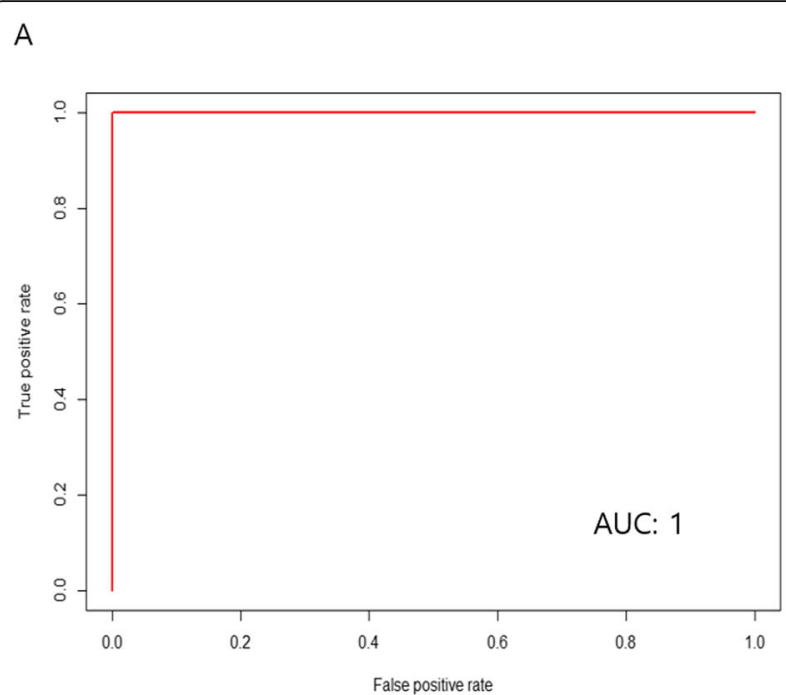

B

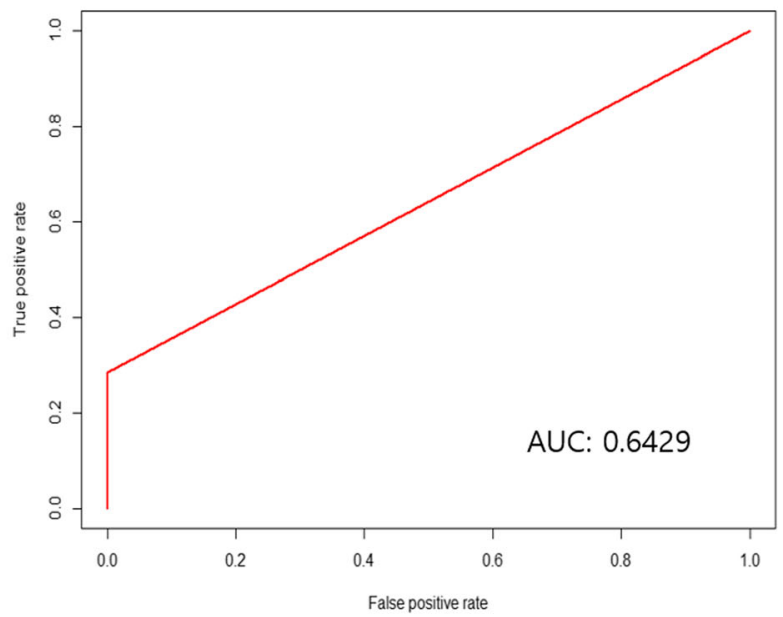

Fig. 4 Receiver operating characteristic (ROC) curve analysis of the biliary tract cancer according to microbiome pattern. a Between normal healthy group and biliary tract cancer group. b Between cholecystitis group and biliary tract cancer group

gut microbiota by analyzing the metagenome analysis from the blood or urine samples, indirectly. The composition alteration may have role in the manifestation of various types of pathology. Microbiome is greatly affected by dietary habits, BMI, and blood lipid level [34]. However, the variables were not analyzed nor matched in this study. Therefore, further study is necessary to overcome the limitations of this study.

There are many published studies addressing the issue of genetic mutations associated with biliary tract cancer. Numerous kinds of genetic mutations had been investigated, and the KRAS, BRAF, TP53, SMAD, and $\mathrm{p} 16$ (INK4) mutations are well known factors for cholangiocarcinoma pathogenesis. And the inflammatory cytokines such as interleukin-6, transforming growth factorbeta, tumor necrosis factor-alpha, and platelet-derived growth factors are also an important factors of cancer pathogenesis [35-38]. Although we were unable to identify the precise genetic mutations associated with the composition of microbiome, the subject was, and remains, very much worthy of the effort because the microbiome is now thought to be the major cause of genetic diversity. As more and more pathophysiological roles for EVs are recognized, it is considered for potential novel targets for treatment. Moreover, modified and engineered extracellular vesicles are likely to have applications in macromolecular drug delivery $[15,17,39]$.

Although the results of this study confirm the encouraging results, further studies are needed. In this study, random sampling was performed to construct a test set and a validation set. However, since only internal validation has been performed, external validation is needed in the future. The results of the study showed that the
AUC converged to 1 , which may be a statistically overfitting error. In this study, the number of cases was relatively small. More cases will need to be analyzed in order to find out the proper bacteria that have diagnostic value among numerous strains. Nevertheless, this study is of great significance in finding hopeful clues about the diagnostic value of microbiomes in the future.

\section{Conclusion}

The microbiome composition of the biliary tract cancer patients and normal healthy adults is found to be different when compared. We were able to develop a biliary tract cancer prediction model from the compositional differences of Bifidobacteriaceae and Pseudomonaceae families and Corynebacteriaceae Corynebacterium, Oxalobacteraceae Ralstonia, and Comamonadaceae Comamonas. Biliary tract cancer patients seem to have altered gut microbiota, which is promising biomarker to differentiate malignancy from the physiology of the normal control group. However, there was no significant difference in microbiome composition between the cholecystitis patients and gallbladder cancer patients. Therefore, further study is necessary to confirm the differences of microbiome composition between biliary tract cancer and benign inflammation. Moreover, the genetic mutation of cancer cells warrant investigation, to confirm the cause and effect.

\section{Abbreviations}

AIC: Akaike information criterion; AUC: Area under the ROC; EV: Extracellular vesicle; OR: Odds ratio; OTU: Operational taxonomic units; PCR: Polymerase chain reaction; ROC: Receiver operating characteristic

Acknowledgements

Not applicable. 


\section{Authors' contributions}

$\mathrm{HL}$ conceived and conducted the study and performed the analyses, interpretation of results, and drafting of the manuscript. WHL performed the analyses and interpretations of the result and assisted in the drafting of the manuscript. HK performed the quality control of data. SKM assisted with the interpretation of the results and drafting of the manuscript. All authors have read and approved the final manuscript.

\section{Funding}

This research was supported by Basic Science Research Program through the National Research Foundation of Korea (NRF) funded by the Ministry of Science, ICT \& Future Planning (2017R1C1B5017902 and NRF-2017M3A9F3047497).

\section{Availability of data and materials}

The datasets generated and/or analyzed during the current study is available from the corresponding author on reasonable request.

\section{Ethics approval and consent to participate}

This study complied with the Declaration of Helsinki and was approved by the Institutional Review Board of Ewha Womans University Mokdong Hospital (2017-07-031). Written informed consent was obtained from al participants.

\section{Consent for publication}

The consent for publication is not applicable because this study does not include any individual details, images, or videos.

\section{Competing interests}

Drs. Huisong Lee, Hyeon Kook Lee, Seog Ki Min, and Won Hee Lee have no competing interests.

\section{Author details}

'Department of Surgery, Ewha Womans University College of Medicine, 1071 Anyangcheon-ro, Yangcheon-gu, Seoul 07985, South Korea. ${ }^{2}$ MD Healthcare Inc., Seoul, South Korea.

Received: 23 July 2019 Accepted: 16 January 2020

Published online: 24 January 2020

\section{References}

1. Sano T, Shimada K, Sakamoto Y, Ojima H, Esaki M, Kosuge T. Prognosis of perihilar cholangiocarcinoma: hilar bile duct cancer versus intrahepatic cholangiocarcinoma involving the hepatic hilus. Ann Surg Oncol. 2008;15:590-9.

2. Ito F, Agni R, Rettammel RJ, Been MJ, Cho CS, Mahvi DM, Rikkers LF, Weber SM. Resection of hilar cholangiocarcinoma: concomitant liver resection decreases hepatic recurrence. Ann Surg. 2008;248:273-9.

3. Jusakul A, Kongpetch S, Teh BT. Genetics of Opisthorchis viverrini-related cholangiocarcinoma. Curr Opin Gastroenterol. 2015:31:258-63.

4. Shukla VK, Singh H, Pandey M, Upadhyay SK, Nath G. Carcinoma of the gallbladder--is it a sequel of typhoid? Dig Dis Sci. 2000:45:900-3.

5. Singh $H$, Pandey $M$, Shukla VK. Salmonella carrier state, chronic bacterial infection and gallbladder carcinogenesis. Eur J Cancer Prev. 1996:5:144.

6. Di Domenico EG, Cavallo I, Pontone M, Toma L, Ensoli F. Biofilm producing Salmonella typhi: chronic colonization and development of gallbladder cancer. Int J Mol Sci. 2017:18:1887. https://doi.org/10.3390/ijms18091887.

7. Koshiol J, Wozniak A, Cook P, Adaniel C, Acevedo J, Azocar L, Hsing AW, Roa JC, Pasetti MF, Miquel JF, et al. Salmonella enterica serovar Typhi and gallbladder cancer: a case-control study and meta-analysis. Cancer Med. 2016:5:3310-235

8. Pandey M, Shukla M. Helicobacter species are associated with possible increase in risk of hepatobiliary tract cancers. Surg Oncol. 2009:18:51-6.

9. Tsuchiya Y, Loza E, Villa-Gomez G, Trujillo CC, Baez S, Asai T, Ikoma T, Endoh K, Nakamura K. Metagenomics of microbial communities in gallbladder bile from patients with gallbladder cancer or cholelithiasis. Asian Pac J Cancer Prev. 2018;19:961-7.

10. Chng KR, Chan SH, Ng AH, Li C, Jusakul A, Bertrand D, Wilm A, Choo SP, Tan $\mathrm{DM}$, Lim $\mathrm{KH}$, et al. Tissue microbiome profiling identifies an enrichment of specific enteric bacteria in Opisthorchis viverrini associated cholangiocarcinoma. EBioMedicine. 2016;8:195-202.

11. Ma P, Pan Y, Li W, Sun C, Liu J, Xu T, Shu Y. Extracellular vesicles-mediated noncoding RNAs transfer in cancer. J Hematol Oncol. 2017;10:57.
12. Kim J, Park WY, Kim NKD, Jang SJ, Chun SM, Sung CO, Choi J, Ko YH, Choi YL, Shim HS, Won JK. Good laboratory standards for clinical nextgeneration sequencing cancer panel tests. J Pathol Transl Med. 2017;51: 191-204.

13. Charbonneau MR, Blanton LV, DiGiulio DB, Relman DA, Lebrilla CB, Mills DA, Gordon JI. A microbial perspective of human developmental biology. Nature. 2016:535:48-55.

14. Kang CS, Ban M, Choi EJ, Moon HG, Jeon JS, Kim DK, Park SK, Jeon SG, Roh TY, Myung SJ, et al. Extracellular vesicles derived from gut microbiota, especially Akkermansia muciniphila, protect the progression of dextran sulfate sodium-induced colitis. PLoS One. 2013:8:e76520.

15. Yoo JY, Rho M, You YA, Kwon EJ, Kim MH, Kym S, Jee YK, Kim YK, Kim YJ. $16 \mathrm{~S}$ rRNA gene-based metagenomic analysis reveals differences in bacteriaderived extracellular vesicles in the urine of pregnant and non-pregnant women. Exp Mol Med. 2016:48:e208.

16. Choi Y, Kwon Y, Kim DK, Jeon J, Jang SC, Wang T, Ban M, Kim MH, Jeon SG, Kim MS, et al. Gut microbe-derived extracellular vesicles induce insulin resistance, thereby impairing glucose metabolism in skeletal muscle. Sci Rep. 2015:5:15878.

17. EL Andaloussi S, Mager I, Breakefield XO, Wood MJ. Extracellular vesicles: biology and emerging therapeutic opportunities. Nat Rev Drug Discov. 2013;12:347-57

18. Lee Y, Park JY, Lee EH, Yang J, Jeong BR, Kim YK, Seoh JY, Lee S, Han PL, Kim EJ. Rapid assessment of microbiota changes in individuals with autism spectrum disorder using bacteria-derived membrane vesicles in urine. Exp Neurobiol. 2017:26:307-17.

19. Akin H, Tozun N. Diet, microbiota, and colorectal cancer. J Clin Gastroenterol. 2014;48(Suppl 1):S67-9.

20. Schag CC, Heinrich RL, Ganz PA. Karnofsky performance status revisited: reliability, validity, and guidelines. J Clin Oncol. 1984;2:187-93.

21. Lee EY, Bang JY, Park GW, Choi DS, Kang JS, Kim HJ, Park KS, Lee JO, Kim YK Kwon $\mathrm{KH}$, et al. Global proteomic profiling of native outer membrane vesicles derived from Escherichia coli. Proteomics. 2007;7:3143-53.

22. Nossa CW, Oberdorf WE, Yang L, Aas JA, Paster BJ, Desantis TZ, Brodie EL, Malamud D, Poles MA, Pei Z. Design of 165 rRNA gene primers for 454 pyrosequencing of the human foregut microbiome. World J Gastroenterol. 2010;16:4135-44.

23. Caporaso JG, Kuczynski J, Stombaugh J, Bittinger K, Bushman FD, Costello EK, Fierer N, Pena AG, Goodrich JK, Gordon Jl, et al. QIIME allows analysis of high-throughput community sequencing data. Nat Methods. 2010;7:335-6.

24. Lozupone C, Hamady M, Knight R. UniFrac--an online tool for comparing microbial community diversity in a phylogenetic context. BMC Bioinformatics. 2006:7:371.

25. Dobbin KK, Simon RM. Optimally splitting cases for training and testing high dimensional classifiers. BMC Med Genet. 2011:4:31.

26. Grice EA, Segre JA. The human microbiome: our second genome. Annu Rev Genomics Hum Genet. 2012:13:151-70.

27. Marchesi JR, Dutilh BE, Hall N, Peters WH, Roelofs R, Boleij A, Tjalsma H. Towards the human colorectal cancer microbiome. PLoS One. 2011;6:e20447.

28. Farrell JJ, Zhang L, Zhou H, Chia D, Elashoff D, Akin D, Paster BJ, Joshipura K, Wong DT. Variations of oral microbiota are associated with pancreatic diseases including pancreatic cancer. Gut. 2012;61:582-8.

29. Kim HJ, Kim YS, Kim KH, Choi JP, Kim YK, Yun S, Sharma L, Dela Cruz CS, Lee $J S$, Oh YM, et al. The microbiome of the lung and its extracellular vesicles in nonsmokers, healthy smokers and COPD patients. Exp Mol Med. 2017:49: e316

30. Pandey M. Helicobacter species are associated with possible increase in risk of biliary lithiasis and benign biliary diseases. World J Surg Oncol. 2007:5:94.

31. Pandey M, Mishra RR, Dixit R, Jaiswal R, Shukla M, Nath G. Helicobacter bilis in human gallbladder cancer: results of a case-control study and a metaanalysis. Asian Pac J Cancer Prev. 2010;11:343-7.

32. Suarez-Munoz MA, Fernandez-Aguilar JL, Sanchez-Perez B, Perez-Daga JA, Garcia-Albiach B, Pulido-Roa Y, Marin-Camero N, Santoyo-Santoyo J. Risk factors and classifications of hilar cholangiocarcinoma. World J Gastrointest Oncol. 2013;5:132-8

33. Kang DW, Park JG, Ithan ZE, Wallstrom G, Labaer J, Adams JB, KrajmalnikBrown R. Reduced incidence of Prevotella and other fermenters in intestinal microflora of autistic children. PLoS One. 2013:8:e68322.

34. Quigley EM, Abu-Shanab A, Murphy EF, Stanton C, Monsour HP Jr. The metabolic role of the microbiome: implications for NAFLD and the metabolic syndrome. Semin Liver Dis. 2016;36:312-6. 
35. Maemura K, Natsugoe S, Takao S. Molecular mechanism of

cholangiocarcinoma carcinogenesis. J Hepatobiliary Pancreat Sci. 2014;21:

754-60.

36. Ross JS, Wang K, Gay L, Al-Rohil R, Rand JV, Jones DM, Lee HJ, Sheehan CE, Otto GA, Palmer G, et al. New routes to targeted therapy of intrahepatic cholangiocarcinomas revealed by next-generation sequencing. Oncologist. 2014;19:235-42

37. Putra J, de Abreu FB, Peterson JD, Pipas JM, Mody K, Amos Cl, Tsongalis GJ, Suriawinata AA. Molecular profiling of intrahepatic and extrahepatic cholangiocarcinoma using next generation sequencing. Exp Mol Pathol. 2015;99:240-4

38. Lee CH, Wang HE, Seo SY, Kim SH, Kim IH, Kim SW, Lee ST, Kim DG, Han MK, Lee SO. Cancer related gene alterations can be detected with nextgeneration sequencing analysis of bile in diffusely infiltrating type cholangiocarcinoma. Exp Mol Pathol. 2016;101:150-6

39. Hsiao EY, McBride SW, Hsien S, Sharon G, Hyde ER, McCue T, Codelli JA, Chow J, Reisman SE, Petrosino JF, et al. Microbiota modulate behavioral and physiological abnormalities associated with neurodevelopmental disorders. Cell. 2013;155:1451-63.

\section{Publisher's Note}

Springer Nature remains neutral with regard to jurisdictional claims in published maps and institutional affiliations.

Ready to submit your research? Choose BMC and benefit from:

- fast, convenient online submission

- thorough peer review by experienced researchers in your field

- rapid publication on acceptance

- support for research data, including large and complex data types

- gold Open Access which fosters wider collaboration and increased citations

- maximum visibility for your research: over $100 \mathrm{M}$ website views per year

At BMC, research is always in progress.

Learn more biomedcentral.com/submissions 